\title{
Diagnostics for the knowledge: the case of the tower of Palazzo Termine Pietragliata in Palermo (Italy) Caterina Gattuso ${ }^{a}$, Federica Fernandez ${ }^{\mathbf{b}}$, Massimiliano Marafon Pecoraro, Anna Maria Palermo \\ ${ }^{a}$ Università della Calabria, Rende (CS), Italy, caterina.gattuso@unical.it, ${ }^{b}$ Università degli Studi di Palermo, Palermo, Italy, fernandez.arch@gmail.com.
}

\begin{abstract}
Developing a methodological approach is crucial to assess the state of conservation of a monument, starting from the overall analysis, progressively moving from small to medium scale and reaching the grand scale at the end. Considering the territory, causes are investigated in order to understand the propensities, as well as considering a single monument, evidences are carefully read to assess the overall conditions, that will be lately detailed by the analysis on materials and their degradation.

The object of this study is the tower of the Palazzo Pietragliata in Palermo, one of the most important examples of the late Gothic civil architecture in Sicily.

The Palace, built in 1473 by Prince of Baucina, possesses an imposing crenellated tower, whose two levels are connected by an original internal staircase "cargol" like, an extraordinary example of the influence of Catalan Gothic architecture in Sicily.

Some micro samples were taken from the tower structure, made entirely of blocks of biocalcarenite, on which diagnostic investigations were performed in the laboratory.

In the context of a more comprehensive interdisciplinary study, this paper illustrates the specific results of the micro analysis conducted in the laboratory and particularly those made through the use of the SEM and biological investigations, also paying attention to the pathologies detected on the battlements of the tower. These forms of decay, for their variety, represent a significant example that allows to properly illustrate the adopted study method.
\end{abstract}

Keywords: tower, conservation, decay, diagnostics.

\section{Introduzione}

Palazzo Pietragliata, elegante dimora situata nel centro storico di Palermo, nonostante i vari interventi subiti nel tempo, rappresenta una delle più importanti testimonianze dell'architettura civile tardo medievale. Di particolare interesse è la caratteristica torre merlata, oggetto dello studio, che oltre ad essere un vero capolavoro architettonico tardogotico filologicamente integro è stata, per molto tempo data la sua altezza, un punto di riferimento persino per le navi che arrivavano nel porto di Palermo. Per dare un contributo diretto alla valorizzazione del palazzo è stato svolto uno specifico studio conoscitivo sviluppando un piano diagnostico interdisciplinare. In particolare dopo aver contestualizzato il manufatto nel suo contesto urbano l'attenzione si concentra sul manufatto che viene analizzato in dettaglio seguendo un percorso organizzato per fasi che prevede la ricostruzione del quadro anamnestico corredato da un adeguato dossier fotografico, (Gattuso 2014) la descrizione architettonica e dello stato 
di conservazione con riferimento ai materiali costitutivi. Lo studio si completa delle analisi di laboratorio dirette ad acquisire informazioni di dettaglio sulle patologie più diffuse e riscontrate durante la campagna di campionamento appositamente predisposta (Gattuso 2012).

\section{Anamnesi e descrizione architettonica}

Oggetto del presente studio è la torre del Palazzo Termine di Pietratagliata, fatto erigere a Palermo nel rione Seralcadio nel 1473 da Antonio Termine, giureconsulto e protonotaro del regno.

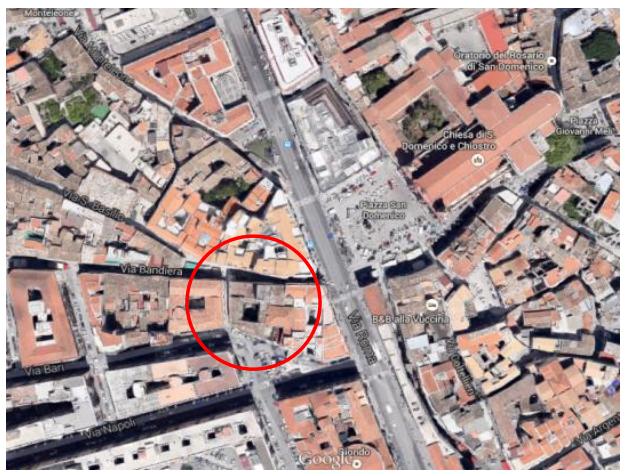

Fig.1 - Localizzazione del Palazzo.

L'edificio si distingue ancora oggi per la facciata principale, merlata e turrita, arricchita dalla presenza di una rara bifora angolare raffinata "prodezza" architettonica tipica del linguaggio tardogotico catalano (Fig. 2).

Il palazzo passa di proprietà nel 1748 , venduto dagli eredi di Antonio Termine a Giovan Battista Marassi, duca di Pietratagliata. Se già nel XVII secolo i Termine avevano realizzato alcuni interventi barocchizzando l'edificio, è soprattutto con i Marassi che il palazzo acquisisce l'importante decorazione interna marcatamente rococò, frutto di un cantiere del 1762 affidato al noto architetto del tempo Giovanni De Frago. Grazie ai restauri di liberazione delle manomissioni d'epoca barocca e di ripristino dei primi decenni del XX secolo, oggi il palazzo rimane una delle più importanti testimonianze dell'architettura civile tardo medievale. Il matrimonio poco più di trenta anni fa tra l'ultimo erede dei Termine, primi committenti del palazzo, con l'ultima erede della famiglia che lo aveva acquistato nel Settecento ha permesso una rara continuità abitativa dell'edificio dalla sua fondazione sino ai giorni nostri. Particolare pregio da questo punta di vista è da attribuire alla torre: se, infatti, alcuni elementi decorativi delle facciate o della decorazione interna sono frutto di un intervento di restauro "leduchiano", questa grazie all'assenza di ripristini, è un vero e proprio capolavoro architettonico tardogotico filologicamente integro.

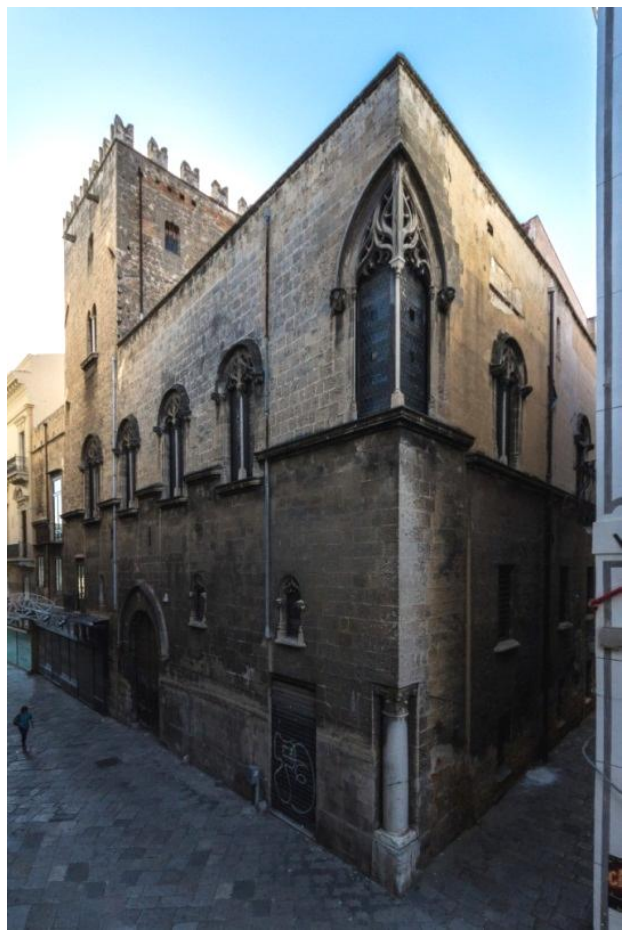

Fig. 2 - Bifora d'angolo.

Le vicende storico architettoniche, venute alla luce grazie a un meticoloso studio nei ricchi archivi delle due uniche famiglie committenti, unitesi recentemente, oltre a consegnarci tutte le informazioni relative ai numerosi cantieri nella fabbrica (da quelli gotici a quelli neogotici passando per gli importanti cantieri barocchi, rococò e neoclassici), ci confermano l'assenza di interventi d'aggiornamento stilistico alla torre, per secoli punto di riferimento persino per le navi che arrivavano nel porto di Palermo, data l'altezza (Fig. 3). 


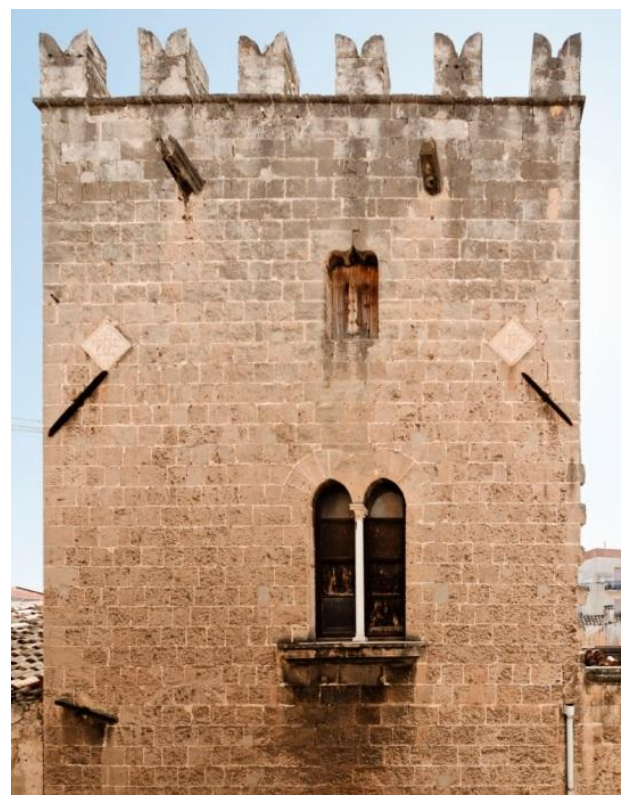

Fig. 3 - La torre.

Alla torre si accede da una scala di grande pregio, una tipica scala a chiocciola sospesa posta all'esterno e addossata. Purtroppo oggi è impossibile distinguerne la funzione aggettante a causa di una superfetazione ottocentesca che ne ingloba la parte basamentale.

Nel 1829, infatti, la realizzazione di una copertura dello scalone principale che dal cortile porta al piano nobile, su progetto di Andrea Gigante, ha irrimediabilmente chiuso alla vista parte della scala a chiocciola che era sospesa proprio sullo scalone e che quindi non era all'interno delle pareti perimetrali della torre stessa. La parte della torre che spicca dal piano nobile della costruzione contiene soltanto due ambienti, uno sovrapposto all'altro, che corrispondono allo spazio delimitato dalle quattro pareti esterne. Quello inferiore è illuminato da una grande bifora che mantiene gli originali sedili per l'affaccio, mentre quello superiore, che ha come copertura il piano di calpestio della terrazza merlata (originario luogo della sentinella) prende luce da una monofora. Al terrazzo di copertura si accede attraverso una scala lignea e una botola con doppio sportello in ferro (Marafon Pecoraro, 2013).

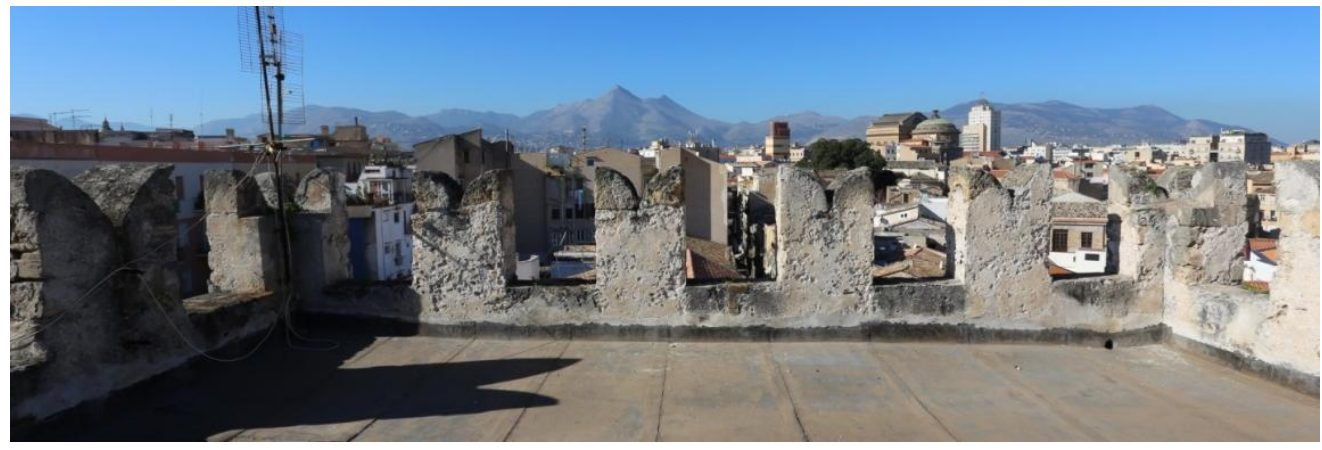

Fig. 4 - Il piano di copertura della torre e i merli.

\section{Lo stato di conservazione}

Lo stato di conservazione generale dell'edificio è discreto, grazie alla continue opere di manutenzione. La torre, in atto, risulta non utilizzata e presenta alcune lesioni verticali nei paramenti murari, dello spessore variabile tra $\mathrm{i}$ 90 e i $60 \mathrm{~cm}$ circa. Il manto di copertura è stato oggetto di un recente intervento di protezione effettuato mediante guaina risvoltata per $20 \mathrm{~cm}$ circa al piede della muratura merlata (Fig. 4).
Lo studio in particolare ha riguardato i merli della torre, parzialmente intonacati nel tempo , che versano attualmente in cattivo stato di conservazione. La torre, così come $\mathrm{i}$ merli, risultano realizzati in biocalcarenite locale, una roccia sedimentaria di origine marina ricca in fossili, caratterizzata da un color giallo paglierino e da una elevata porosità. Si rileva comunque un limitato utilizzo per la costruzione anche di una biocalcarenite più compatta costituita da granulometria degli inerti più fine $\mathrm{e}$ 
minore presenza di fossili. I blocchi maggiormente danneggiati risultano quelli in biocalcarenite del primo tipo, nei quali si rilevano, soprattutto nelle parti più esposte quali gli spigoli, vari fenomeni di alterazione: alveolizzazione con formazione di vuoti profondi anche alcuni centimetri, disgregazione che in alcuni casi si presenta come polverizzazione con perdita di grani al tatto.

La parte sommitale orizzontale presenta uno strato superficiale di incrostazione scura di spessore e consistenza variabili, mista di frequente a patina biologica, costituita da licheni a tallo crostoso di vario tipo: nero, verde-giallo, grigio biancastri.

Nel tempo e, sicuramente, a causa del fatto che il materiale lapideo con cui erano stati realizzati i merli mal resisteva all'esposizione degli agenti atmosferici, sono stati eseguiti vari interventi per integrare le lacune e riconfigurare la forma originale che si andava perdendo, attraverso l'utilizzo di malte con le quali la muratura è stata sarcita, e di intonaci con la funzione di protezione delle superfici in pietra.

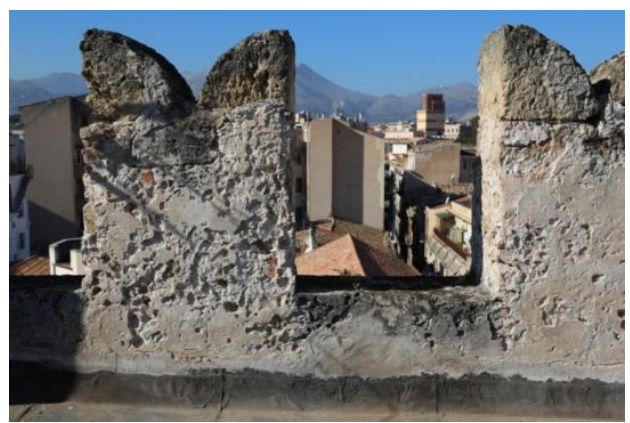

Fig. 5 - Superfici degradate dei merli.

Gli stessi intonaci (si rilevano solo due strati), si presentano oggi in pessimo stato di conservazione: molte parti risultano mancanti e i brani rimasti sono in gran parte distaccati dal substrato, lesionati, decoesi e con frequenti casi di polverizzazione (Fig.6, Fig.7).

Le porzioni di intonaco al piede della muratura presentano anche efflorescenze diffuse, da umidità di risalita per probabile infiltrazione d'acqua nel punto di contatto con il piano di copertura. Si rileva comunque che l'intonaco con cui erano state protette le superfici, realizzato con malta di calce idraulica, era già in origine di scarsa fattura: nell'arriccio si trovano ciottoli di fiume grandi in alcuni casi fino a 15 $\mathrm{mm}$. Sono presenti alcune integrazioni realizzate nel 1980 con una malta a grana fine, molto compatta e rigida che, non avendo avuto un comportamento solidale con la muratura, risulta spesso fratturata (Fernandez, 2007).

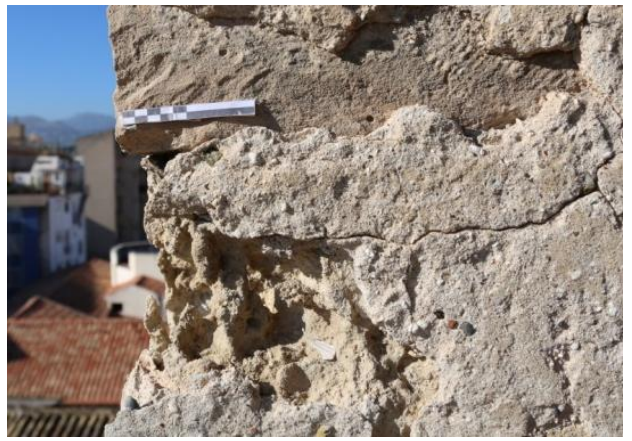

Fig. 6 - Grave alveolizzazione di uno spigolo.
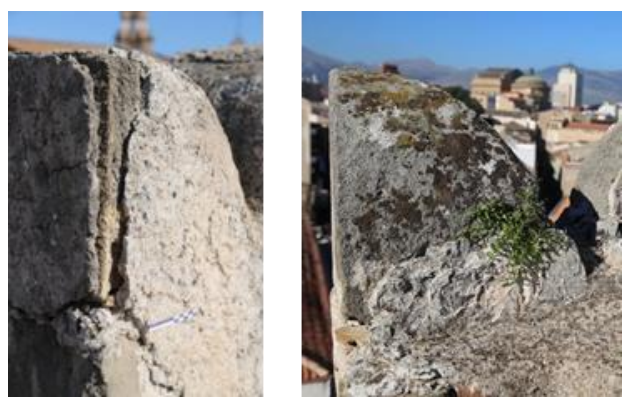

Fig. 7 - Intonaco distaccato e degrado biologico.

\section{Campionamento}

Per acquisire maggiori informazioni sulle patologie rilevate è stato organizzato un piano di campionamento che ha riguardato i merli della torre. Pur avendo effettuato un certo numero di prelievi in vari merli, nel lavoro viene illustrato a titolo esemplificativo un campione che per le sue caratteristiche può essere considerato rappresentativo dello stato di degrado delle superfici intonacate. In particolare è stato individuato e selezionato un campione prelevato in corrispondenza di parti con intonaci in evidente fase di distacco e ricoperti da un denso strato di croste scure (Fig. 8, Fig. 9). 


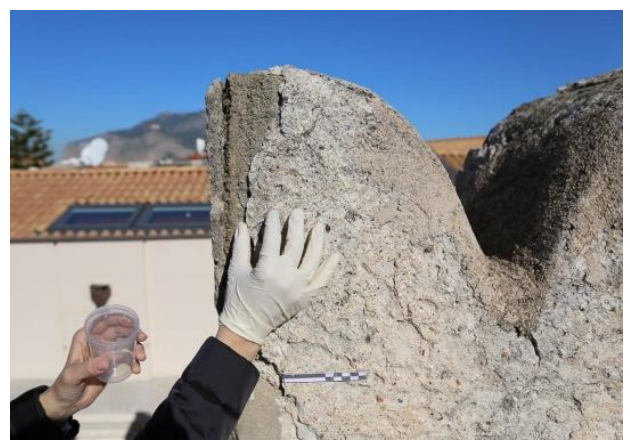

Fig. 8 - Punto di prelievo del campione.

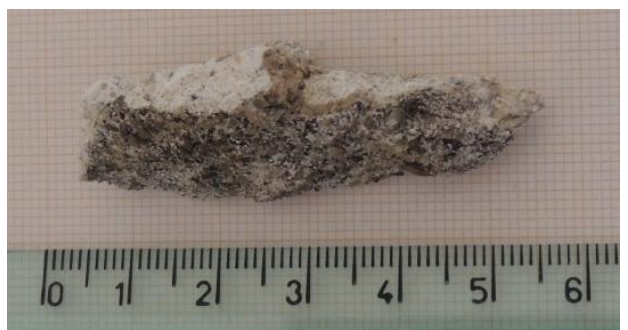

Fig. 9 - Il campione analizzato.

\subsection{Analisi morfologica}

Il campione analizzato, fortemente degradato, è caratterizzato dalla presenza di fratture profonde e risulta essere composto da zone che hanno subito con molta probabilità un processo di ricristallizzazione (Fig. 10, Fig. 10a). Tali zone del campione sono costituite da gruppi di cristalli dalla forma ben definita e dall'abito aciculare (Fig. 10b, Fig.11c).

Inoltre ponendo l'attenzione sulla superficie del campione è stato possibile rilevare ed osservare la presenza di una crosta superficiale (Fig. 12).

\subsection{Analisi chimica}

In relazione alle osservazioni morfologiche si è scelto di condurre l'analisi chimica sia su una porzione della matrice interna del campione che su una porzione di crosta superficiale, con l'obiettivo di identificare differenze nella composizione da imputare ad eventuali fenomeni di alterazione e/o degrado. A tal proposito sono stati acquisiti gli spettri di fluorescenza di entrambe le zone analizzate usando la microsonda EDS unita al microscopio elettronico a scansione (Fig. 13, Fig. 14).
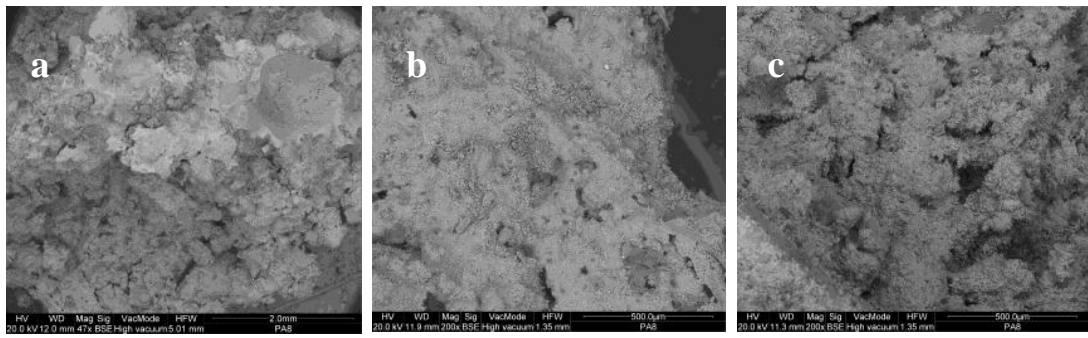

Fig. 10 - Vista d'insieme del campione (a); particolare della matrice con zone ricristallizzate (b, c).
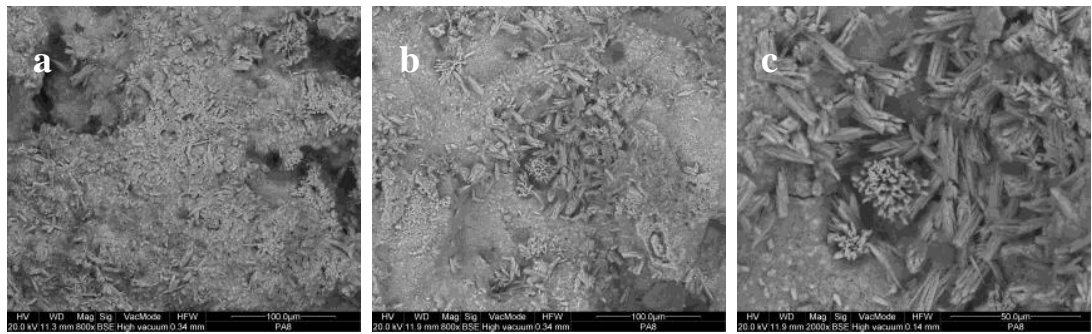

Fig. 11 - Particolare della matrice con evidenti zone ricristallizzate (a); zona ricristallizzata (b); particolare di gruppi di cristalli, ingrandimento 2000X (c). 

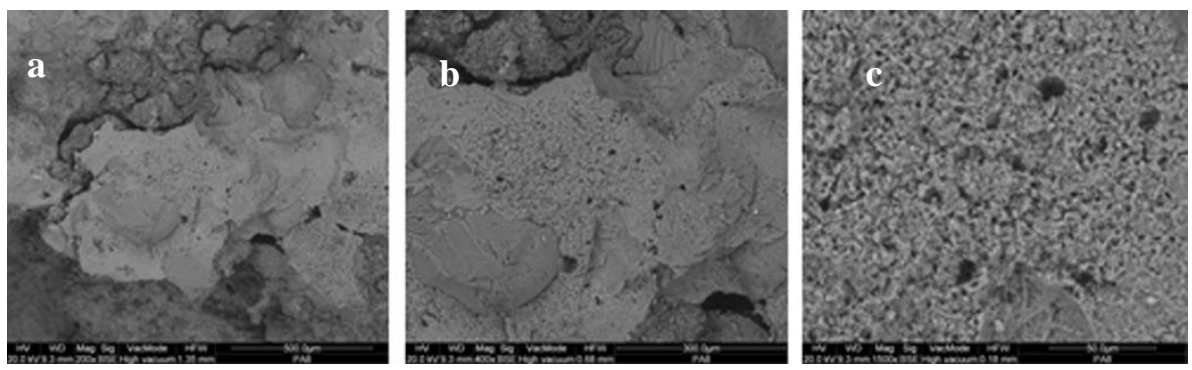

Fig. 12 - Interfaccia matrice interna - crosta superficiale (a, b); particolare della crosta (c).

Dai risultati ottenuti è emerso che la matrice interna è composta quasi interamente da calcite con minime quantità di $\mathrm{Mg}$ e Si e che quindi con molta probabilità corrisponde ad una porzione di intonaco, a conferma delle informazioni ottenute da un esam macroscopico effettuato su campione preliminarmente. L'analisi condotta sulla crosta superficiale pur avendo fornito la medesima composizione chimica della matrice, se ne differenzia per il contenuto maggiore di $\mathrm{Mg}$ e Si e per la presenza di basse quantità di sali $(\mathrm{Na}, \mathrm{Cl})($ Tab. 1).
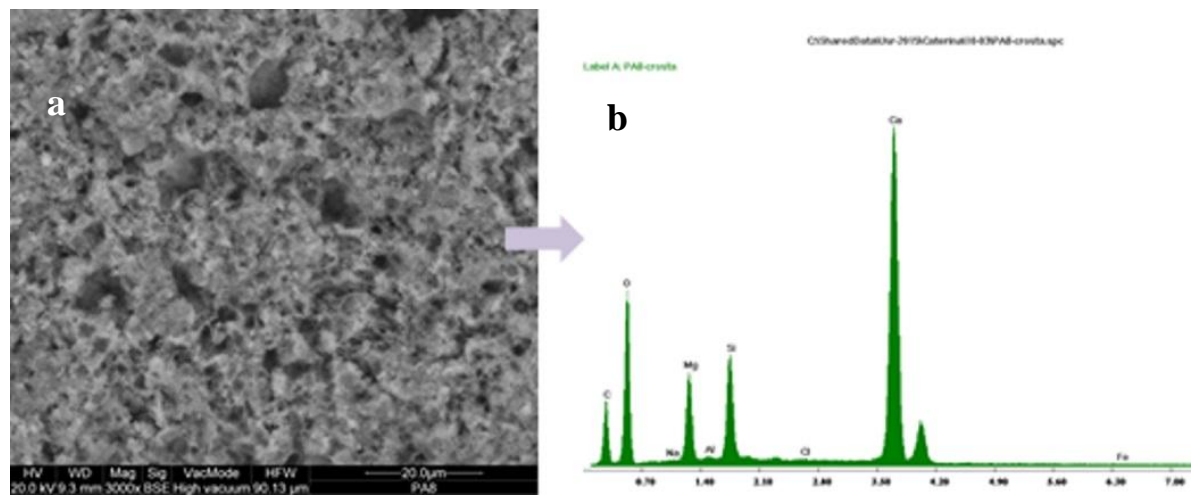

Fig. 13 - Matrice interna su cui è stata effettuata l'analisi chimica (a); Spettro di fluorescenza (b).
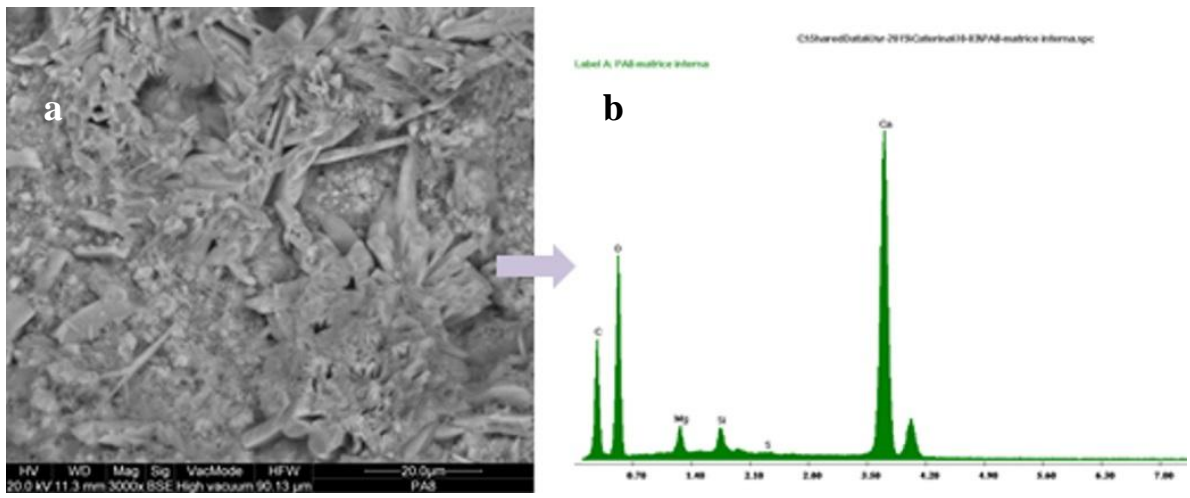

Fig. 14-Crosta superficiale su cui è stata effettuata l'analisi chimica (a); Spettro di fluorescenza (b). 


\begin{tabular}{|c|c|c|c|c|c|c|c|c|c|c|c|}
\hline & a & Mg & I & $\mathbf{S i}$ & $\mathbf{P}$ & $\mathrm{Cl}$ & $\mathbf{S}$ & $\mathrm{Ca}$ & $\mathbf{T i}$ & $\mathrm{Fe}$ & \\
\hline w & \multicolumn{11}{|c|}{ Percen tu a le $(\% 6)$} \\
\hline &.- & & $\ldots$ & & $\ldots$ & $\ldots$ & 0.27 & & $\ldots$ & $\ldots$ & \\
\hline Crosta superficiale & 0.41 & 13.57 & 0.66 & 15.06 & $\ldots 0$ & 0.20 & $\ldots$ & 69.47 & $\ldots$ & 0.62 & \\
\hline
\end{tabular}

Tab. 1 - Elementi maggiori espressi in wt \% relativi alla matrice interna e alla crosta superficiale.

\subsection{Analisi biologica}

La presenza piuttosto diffusa di patine biologiche sulle superfici dei merli ha indotto a campionare e caratterizzare anche le specie biologiche esistenti. I prelievi sono stati analizzati sia mediante l'osservazione diretta seguita, sia attraverso la visualizzazione allo stereomicroscopio e, infine, mediante l'utilizzo di tamponi sterili che sono stati opportunamente trattati ed analizzati in laboratorio (Fig 15).

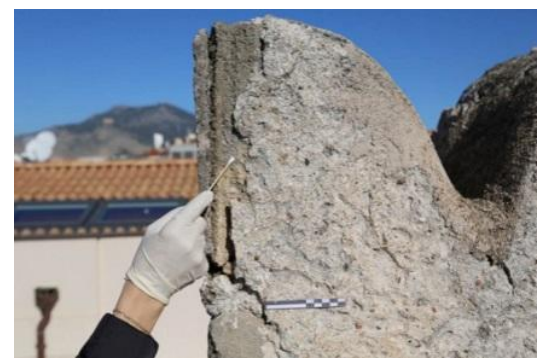

Fig. 15 - Il prelievo con tampone.

Anche in questo caso, dei prelievi effettuati si illustrano i risultati che sono stati rilevati come più significativi. Innanzitutto è stata individuata la presenza di un lichene epilitico crostoso (Fig. 16) con evidente presenza di corpi fruttiferi (apoteci), appartenente al genere Caloplaca.

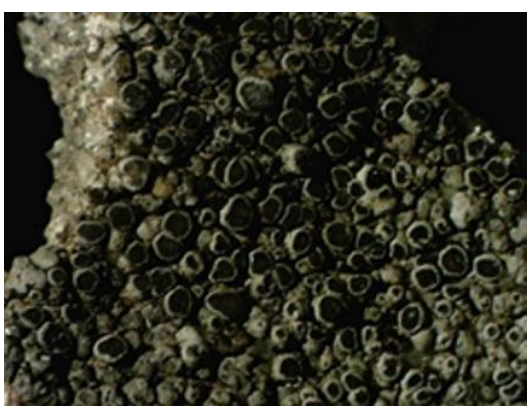

Fig. 16 - Caloplaca sp.

I tamponi sono stati strisciati sul terreno MEA (Malt Extract Agar) e inseriti in cella a $25^{\circ} \mathrm{C}$ per circa 10 giorni.

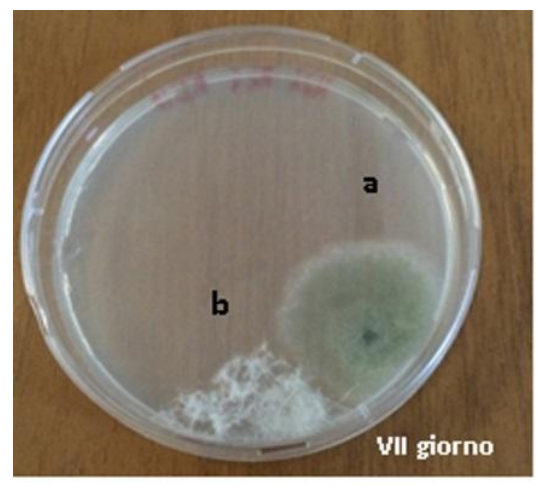

Fig. 17 - Capsula Petri con le 2 colonie fungine.
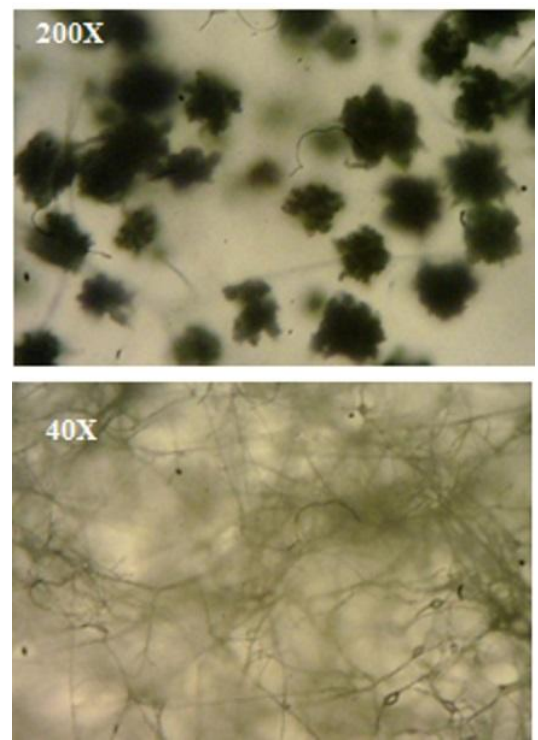

Fig. 18 - Cladosporium sp. Conidiofori (a), Fusarium sp. intreccio di ife (b).

Dall'esame con lo stereomicroscopio e il microscopio ottico, le due colonie sviluppate (Fig. 18) appartengono a due generi fungini:

a) Cladosporium sp.

b) Fusarium sp., 
Nelle immagini si nota la presenza dei conidiofori tipici del genere Cladosporium e l'intreccio ifale del genere Fusarium.

Per quanto riguarda l'approccio biologico è stato possibile mettere in evidenza un lichene del genere Calopalca, la cui presenza è spesso riportata su calcari duri esposti al sole (Nimis, 1992) e due generi fungini comunemente ritrovati nell'aria (Fig. 16). La loro presenza sui materiali lapidei è molto frequente e sia in forma epilitica che endolitica possono giocare un ruolo importante nel loro degrado, come riportato ampiamente in letteratura (Sterflinger, 2010).

\section{Conclusioni}

Per predisporre un corretto progetto di restauro è indispensabile svolgere indagini conoscitive che consentano di definire lo stato di conservazione del manufatto in esame.

Occorre a tal fine pianificare e svolgere un piano diagnostico adeguato che consenta di gestire la complessità della situazione in maniera da comprendere e definire gli approfondimenti necessari. Con riferimento al Palazzo di Pietratagliata la struttura conoscitiva impostata ha consentito di definire le principali fasi del piano diagnostico. Nello specifico è stato tracciato il profilo amnestico dell'edificio che ha consentito di evidenziarne l'importanza dal

punto di vista storico-architettonico essendo un raro esempio di stile tardogotico catalano.
Secondo la procedura metodologica utilizzata è stato delineato lo stato di conservazione con specifica attenzione alla torre merlata caratterizzata dalla presenza diffusa di varie patologie, da ricondurre anche al contesto ambientale in cui è inserita. Sulla base dell'analisi del degrado è stato quindi predisposto un piano di campionamento che ha consentito di prelevare dei piccoli campioni di intonaco in evidente stato di degrado già in fase di distacco. Un campione tra quelli prelevati, selezionato come rappresentativo, è stato caratterizzato in laboratorio, in particolare sono state svolte delle analisi al microscopio elettronico a scansione (SEM) che hanno consentito di osservarne la parte degradata e quella integra. Inoltre, utilizzando un sistema di microanalisi in dispersione di energia (EDS) è stato anche possibile svolgere un'analisi composizionale.

Infine, a completamento, su un campione ottenuto mediante prelievo con tampone sterile è stata effettuata un'analisi per individuare e caratterizzare il degrado biologico.

Lo studio, pur se suscettibile di ulteriori e maggiori approfondimenti, è comunque impostato nelle sue fasi principali e si pone come una struttura di base articolata e completa che permette di definire successivi campi di indagine da sviluppare per acquisire e le informazioni da inserire a completamento nella cartella clinica digitale del bene.

\section{References}

Fernandez, F. (2007), Alterazione e degrado dei materiali lapidei naturali-Fenomeni, cause, riconoscimento e classificazione, UniService, Firenze, pp. 1-145.

Gattuso, C. (2014),. Science and knowledge, an essential synergy to protect and enhance Cultural Heritage. Diagnosis for the conservation and valorization of Cultural Heritage, vol. 5, Aracne Editrice, Roma, pp. 33-38.

Gattuso, C., Gattuso, P., Cozza, R. \& F. Villella (2012), La conoscenza per il restauro e la conservazione: Il ninfeo di Vadue a Carolei e la fontana nuova a Lamezia Terme, Ed. Franco Angeli, Milano, pp. 1-80.

Marafon Pecoraro, M., Palazzotto, P., \& M. Vesco (2013), Palazzo Termine Pietratagliata tra tardogotico e neostili. Archivi, cantieri, protagonisti a Palermo, 40due Edizioni, Palermo

Nimis, P.L., Pinna, D. \& O. Salvadori (1992), Licheni e conservazione dei monumento, Ed. CLUEB, Bologna.

Sterflinger, K. (2010), Fungi: Their role in deterioration of cultural heritage, Fungal Biology Reviews (24, 1-2), pp. 47-55. 